\title{
Label-free electrochemical detection of an Entamoeba histolytica antigen using cell-free yeast-scFv probes ${ }^{\dagger}$
}

\author{
Yadveer S. Grewala ${ }^{\mathrm{a}}$ Muhammad J. A. Shiddiky ${ }^{\mathrm{a}}$, Sean A. Gray ${ }^{\mathrm{b}}$, Kris M. Weigel ${ }^{\mathrm{b}, \mathrm{c}}$, Gerard \\ A. Cangelosi ${ }^{\mathrm{b}, \mathrm{c}}$, and Matt Trau ${ }^{\mathrm{a}}$ \\ Muhammad J. A. Shiddiky: m.shiddiky@uq.edu.au; Matt Trau:m.trau@uq.edu.au \\ ${ }^{a}$ Centre for Biomarker Research and Development, Australian Institute for Bioengineering and \\ Nanotechnology, The University of Queensland, Brisbane, QLD 4072, Australia. Tel: \\ +61-7-33464178. Fax: +61-7-33463973 \\ bSeattle Biomedical Research Institute, Seattle, WA 98117, USA
}

\begin{abstract}
Inexpensive, simple and quick detection of pathogen antigens in human samples is a key global health objective. Limiting factors include the cost and complexity of diagnostic tests that utilize antibody probes. Herein, we present a method for label-free electrochemical detection of a protein from the enteric pathogen Entamoeba histolytica using cell-free yeast-embedded antibody-like fragments (yeast-scFv) as novel affinity reagents.
\end{abstract}

The waterborne pathogen Entamoeba histolytica (E. histolytica) is a prevalent but treatable disease that is estimated to cause 100,000 deaths annually in developing countries. ${ }^{1}$ Prior detection techniques relied on microscopy and serology. Microscopy tests cannot distinguish between $E$. histolytica and closely related non-pathogenic commensals that also occur in stool, most notably E. dispar. Serological tests, which detect serum antibodies against $E$. histolytica, cannot distinguish between past and current infection. ${ }^{1,2}$ For these reasons molecular tests, such as enzyme-linked immunosorbent assay (ELISA), have become preferred diagnostics to identify $E$. histolytica infection, due to their specificity and sensitivity. ${ }^{2}$

ELISA-based methods have the limitation of being reliant on highly specific affinity reagents, typically monoclonal antibodies (mAbs). The considerable time and resources required for the generation of $\mathrm{mAbs}$ is a bottleneck in biomedical research and the development of new diagnostic tests. Potential alternatives to mAbs are fragments of antibodies known as single-chain variable fragments (scFv). ${ }^{3}$ In contrast to traditional mAbs derived from vertebrate animals, scFv reagents can be cheaply and rapidly selected from yeast-display and other libraries. In yeast display, libraries of genetically engineered Saccharomyces cerevisiae cells display on their surfaces diverse scFv molecules derived from human mRNA. By using fluorescence-activated cell sorting (FACS), it is possible in a 2-3 week process to screen yeast display libraries for clones that bind specifically to antigens. If needed, affinity enhancement can be completed in another 3-4 weeks. Biosensors incorporating scFvs for specific antigen detection have been described. ${ }^{4-11}$

\footnotetext{
$\dagger$ Electronic Supplementary Information (ESI) available: Experimental details and DLS spectrum. See DOI: 10.1039/b000000x/

Correspondence to: Muhammad J. A. Shiddiky, m. shiddiky@uq. edu . au; Matt Trau, m. trau@uq. edu . au.

${ }^{c}$ Current address: Department of Environmental and Occupational Health Sciences, The University of Washington, Seattle, WA 98195-9472, USA.
} 
Although many useful scFvs have been reported, most scFvs derived from display libraries perform unsatisfactorily in solution. ${ }^{12}$ Like natural antibodies, antibody-like fragments culled from display libraries are products of selection. Yeast-displayed $\mathrm{scFv}$ are selected by FACS for affinity and stability when bound via Aga1-Aga2 linkages to yeast cell walls. Unfortunately, $\mathrm{scFv}$ that have excellent activity on yeast surfaces usually lose their activity in solution, an environment for which they were not selected. This problem has limited the penetration of $\mathrm{scFv}$ into diagnostic practice.

Affinity reagents composed of lyophilized whole yeast cells with displayed scFv (yeast$\mathrm{scFv}$ ) have been shown to be viable, cheap and quick alternatives to generating mAbs or soluble $\mathrm{scFv}$ for use in immunoassays. ${ }^{13}$ In contrast to soluble scFv generated by recombinant expression systems, yeast-scFv maintains the antibody-like fragments in the yeast surface environments in which they were selected by FACS to function. They are robust and renewable reagents that can be produced in vast quantities at low cost. However these whole-cell reagents are insoluble and too large for many diagnostic applications. Moreover, they required the use of labelled polyclonal antibodies to detect antigen binding to the yeast-scFv particles. ${ }^{13}$ Although it was not necessary that the detection antibodies be highly specific to the antigen (monoclonal specificity was conferred by the yeast-scFv reagent), the requirement for a traditional animal-derived detection antibody diluted the benefits of using yeast-scFv.

To enable the use of yeast-scFv affinity reagents as full and practical alternatives to traditional antibodies, the current report describes two new approaches. First, cell-free yeast$\mathrm{scFv}$ reagents were generated by mechanical fragmentation of whole yeast-scFv cells, followed by combined mechanical and affinity-based purification of semi-soluble cell wall fragments bearing displayed $\mathrm{scFv}$. As with whole-cell yeast-scFv reagents, we hypothesized that $\mathrm{scFv}$ associated with cell wall fragments would retain the stability and functionality for which they were selected. Second a label-free electrochemical (EC) approach was used to detect antigen binding to cell-free yeast-scFv, without the need for animal-derived detection antibodies. These approaches were combined to generate a specific, sensitive sensor for a candidate $E$. histolytica cyst protein.

The principle of faradaic electrochemical impedance spectroscopy (F-EIS) was used for label-free EC detection. This is one of the most effective methods for the label-free detection of biomolecules and for probing the build-up of the biomaterials sensing film on the electrodes. ${ }^{14}$ In F-EIS, the successful capture and detection of the biomolecule of interest, here an E. histolytica protein, is observed as a change in the capacitance and interfacial electron transfer resistance of a conductive or semiconductive electrode modified with the scFv capture probe. ${ }^{14} \mathrm{~A}$ typical shape for a F-EIS spectrum at a disk macroelectrode, presented in the form of a Nyquist plot (e.g., $Z^{\prime}$ versus $Z^{\prime \prime}$ at variable frequencies, where $Z^{\prime}$ and $Z^{\prime \prime}$ are the real and imaginary components), includes a semicircle region lying on the $Z^{\prime}$ axis followed by a straight line. ${ }^{15}$ The semicircle portion is observed at higher frequencies and corresponds to the electron-transfer-limited process. ${ }^{14,15}$ The diameter of the semicircle corresponds to the electron-transfer resistance at the electrode surface, $R_{\mathrm{et}}$. Therefore, the build-up of an immunosening layer and scFv-antigen complex formation can be followed by F-EIS, where the change in impedance of the electrode surface and its interface to the electrolyte solution containing a redox probe $\left(\right.$ e.g., $\left.\left[\mathrm{Fe}(\mathrm{CN})_{6}\right]^{3-/ 4-}\right)$ at different stages of the immunosensor fabrication is measured in the form of its $R_{\mathrm{et}}$.

Fig. 1A outlines the approach for using a cell-free yeast-scFv reagent in the label-free detection of an E. histolytica antigen. Briefly, a biolayer on a Au surface is formed with biotinylated BSA (bio BSA). Multivalent streptavidin is used to link the bio BSA to a biotinylated anti-human influenza hemagglutinin tag antibody (bio anti-HA). This complex 
is used to capture cell-free yeast-scFv fragments by virtue of an HA antigen tag cloned into the recombinant $\mathrm{scFv}$ construct. The bound yeast-scFv fragment is then used to capture the target E. histolytica protein EHI_115350, called '350' in this report (additional details available in $\left.\mathrm{ESI}^{\dagger}\right) .{ }^{13}$ To assure that the fragments were small enough to be useable in the bioassay, dynamic light scattering (DLS) measurements of the yeast-scFv fragments after filtration through a $0.1 \mu \mathrm{m}$ filter was performed. The DLS data confirmed that fragments existed in the sub-100 nm size range (Fig. S1, $\mathrm{ESI}^{\dagger}$ ), allowing for attachment of yeast-scFv fragments to bio anti-HA.

The attachment of the bio BSA, streptavidin, yeast-scFv, and E. histolytica antigens on the gold electrode was followed by the F-EIS and differential pulse voltammetry (DPV) (Fig. $1 \mathrm{~B}$ and C). The BSA layer, in combination with subsequent attachment of streptavidin and antibody, act as a barrier for the interfacial electron transfer reaction of the $\left[\mathrm{Fe}(\mathrm{CN})_{6}\right]^{3-/ 4-}$ process, resulting in an increase in $R_{\mathrm{et}}$. When we monitored $R_{\mathrm{et}}$ generated by the $\left[\mathrm{Fe}(\mathrm{CN})_{6}\right]^{3-/ 4-}$ process before and after protein binding, there was a clear correlation between the presence of the target $E$. histolytica antigen and the increases of the $R_{\text {et }}$ (Fig. 2). The presence of the $E$. histolytica antigen appears to further block the $\left[\mathrm{Fe}(\mathrm{CN})_{6}\right]^{3-/ 4-}$ process from accessing the electrode surface effectively. The bare Au electrode (i) gave rise to the lowest semicircle domain indicating the fastest electron transfer (e.g., the lowest $\left.R_{\mathrm{et}}\right)$ followed by bio BSA/streptavidin-coated Au electrode (ii), bio anti-HA (iii), yeast-scFv antibody immobilized electrode (iv), and target antigen $(100 \mathrm{pg} / \mathrm{mL})(\mathrm{v})$. These results indicate successful stepwise binding of biomolecules on the sensor.

The risk of false-positive responses for targets at low concentrations is well-known when using a detection technique based on attenuation of the interfacial electron transfer reaction of a redox process. To assess this risk, a series of parallel independent DPV measurements were conducted. Fig. 2B shows the differential pulse profiles, where the peak current decreases in response to increasing the hindrance of the interfacial electron transfer reaction of the $\left[\mathrm{Fe}(\mathrm{CN})_{6}\right]^{3-/ 4-}$ process. This is in line with the results observed in Faradaic EIS by the same electrode.

The specificity of the immunosensor towards ' 350 ' antigen was examined by attempting to capture an antigen that was non-specific to the immobilized yeast-scFv probes. The Jacob protein of $E$. histolytica was previously shown not to bind with 350 -specific yeast-scFv. ${ }^{13}$ Non-specific binding of the Jacob protein to the cell-free yeast-scFv probes was evaluated by using F-EIS and DPV measurements. A relatively high concentration of the Jacob protein $(500 \mathrm{pg} / \mathrm{mL})$ was used to maximize detection of background binding. A slight increase in the impedance (e.g., $R_{\mathrm{et}}$ value) and decrease in the DPV peak current (Fig. 3A(i) and 3B (i)) were seen for the bio BSA/streptavidin/bio anti-HA/yeast-scFv/Jacob-coated electrode compared to that of the bio BSA/streptavidin/bio anti-HA/yeast-scFv-coated electrode (dotted line in Fig. 3A). A likely explanation for this observation was that the traditional BSA blocking method used in this approach could allow some degree of non-specific adsorption of the Jacob antigen. However, the signal changes for $500 \mathrm{pg} / \mathrm{mL}$ Jacob antigen were smaller than that produced by the target ' 350 ' antigen even when the latter was present at 50 -fold lower concentration $(10 \mathrm{pg} / \mathrm{mL})$. This indicates that label-free immunosensing with a cell-free yeast-scFv reagent has specificity toward the target antigen.

To further assess whether the yeast cell wall fragment may contribute to non-specific adsorption of antigens, a second yeast-scFv fragment that is specific to an orthogonal $E$. histolytica antigen (named '780') (hereafter referred to as yeast-scFv-2) was used as a control. Fig. 3A(ii) and 3B(ii) show the impedance and DPV responses obtained for $500 \mathrm{pg} /$

\footnotetext{
$\dagger$ Electronic Supplementary Information (ESI) available: Experimental details and DLS spectrum. See DOI: 10.1039/b000000x/
} 
$\mathrm{mL}$ ' 350 ' antigen at yeast-scFv-2 coated electrode. Compared to the response for $10 \mathrm{pg} /$ $\mathrm{mL}_{5}$ '350' antigen at cognate yeast-scFv coated sensing layer (Fig. 3A(iv) and 3B (iv), the use of yeast-scFv-2 significantly decreased the sensor impedance $\left(e . g\right.$., lower $\left.R_{\mathrm{et}}\right)$ and increased the DPV peak current, even when a 50-fold higher concentration of ' 350 ' antigen was used. Thus, the non-cognate yeast- $\mathrm{scFv}-2$ reagent failed to capture ' 350 ' antigen with the same efficiency as the cognate yeast-scFv reagent. It was also noted that a similar $R_{\text {et }}$ and peak current values to Jacob antigen were observed, which might indicate a low level of nonspecific adsorption of antigen on yeast cell wall.

To assess the background noise, an assay was performed which excluded the yeast-scFv layer (Fig. 3A(iii) and 3B (iii)). This assay detected non-specific interaction and adsorption of the '350' antigen on the bio BSA/streptavidin/bio anti-HA electrode. The $R_{\text {et }}$ and DPV peak currents without yeast-scFv are comparable to the $R_{\mathrm{et}}$ and peak currents to the bio antiHA surface (see Fig. 2(iii) versus Fig. 3(iii)). These results demonstrate that detection of ' 350 ' antigen by the label-free assay is a function of specific interactions between the cellfree yeast-scFv reagent and the antigen, and not to non-specific interactions between the antigen and other components of the assay.

To assess the dynamic range for detection of ' 350 ' antigen, the percent changes in DPV current responses (see $\mathrm{ESI}^{\dagger}$ for calculation details) were measured before and after incubation of the yeast-scFv-modified electrode with designated concentration of ' 350 ' antigens (10 pg/mL to $10 \mathrm{ng} / \mathrm{mL})$. Signals increased quantitatively with antigen concentration (Fig. 4). The RSD (relative standard deviation) of $4.02 \%(n=3)$ was found in inter-assay signals. Saturation of the Au surface begins to occur at $2500 \mathrm{pg} / \mathrm{mL}(3.5 \mu \mathrm{M})$ of 350 antigen. Detection of the antigen above background was confirmed down to $100 \mathrm{pg} / \mathrm{mL}$ $(558 \mathrm{pM})$. This concentration of antigen was similar to the limit of detection of the same antigen by a whole yeast-scFv probe used in combination with fluorescently labelled polyclonal signal antibody, as described previously. ${ }^{13}$ A precise lower limit of detection was not determined in this experiment.

In conclusion, we have demonstrated a label and cell-free electrochemical immunoassay towards an E. histolytica antigen using antibody fragments embedded in yeast cell wall fragments as capture probes. The principle of this cell-free yeast-scFv based assay is not limited to E. histolytica 350 antigen, as the immunosensor could be fabricated towards any protein-based disease marker by selecting new yeast-scFv against those antigens. We did not determine whether the level of sensitivity afforded by our method exceeds flow cytometry based assays. ${ }^{13}$ However, key architectural improvements were made, including: (i) avoiding use of secondary antibodies and (ii) utilising yeast-scFv cell wall fragments. Noncovalent adsorption of antibody on bio-BSA layer also circumvents the need for chemical activation step required for covalent bindings, greatly simplifying the assay protocol and reducing analysis time. The electrochemical readout is also relatively low cost, and thus the proposed method has excellent potential to translate into diagnostic applications.

\section{Supplementary Material}

Refer to Web version on PubMed Central for supplementary material.

\section{Acknowledgments}

This work was supported by the ARC DECRA (DE120102503) and NIH Grant, USA (U01AI082186-01). Darby M. Kozak is acknowledged for his comments on the manuscript. 


\section{Notes and references}

1. Tanyuksel M, Petri WA. Clin Microbiol Rev. 2003; 16:713-729. [PubMed: 14557296]

2. Haque R, Petri WA Jr. Arch Med Res. 2006; 37:272-275.

3. Holliger P, Hudson PJ. Nat Biotechnol. 2005; 23:1126-1136. [PubMed: 16151406]

4. Backmann N, et al. PNAS. 2005; 102:14587-14592. [PubMed: 16192357]

5. Torrance L, et al. J Virol Methods. 2006; 134:164-170. [PubMed: 16427706]

6. Shen Z, Yan H, Zhang Y, Mernaugh RL, Zeng X. Anal Chem. 2008; 80:1910-1917. [PubMed: 18290668]

7. Joung CK, et al. Sensor Actuat B:Chem. 2012; 161:824-831.

8. Shiddiky MJA, et al. Biosens Bioelectron. 2012; 38:132-137. [PubMed: 22705407]

9. Shang Y, Mernaugh R, et al. Anal Chem. 2012; 84:8164-8170. [PubMed: 22934911]

10. Heo NS, et al. Sensors. 2012; 12:10097-10108. [PubMed: 23112590]

11. Zeng X, et al. Anal Bioanal Chem. 2011; 402:3027-3038. [PubMed: 22159424]

12. Miller KD, et al. Protein Expres Purif. 2005; 42:255-267.

13. Gray SA, et al. PLoS ONE. 2012; 7:e32042. [PubMed: 22363793]

14. Katz E, Willner I. Electroanalysis. 2003; 15:913-947.Zhou M, Dong S. Acc Chem Res. 2011; 44:1232-1243. [PubMed: 21812435]

15. Bard, AJ.; Faulkner, LR. Electrochemical Methods: Fundamentals and Applications. John Wiley \& Sons; 2000. 

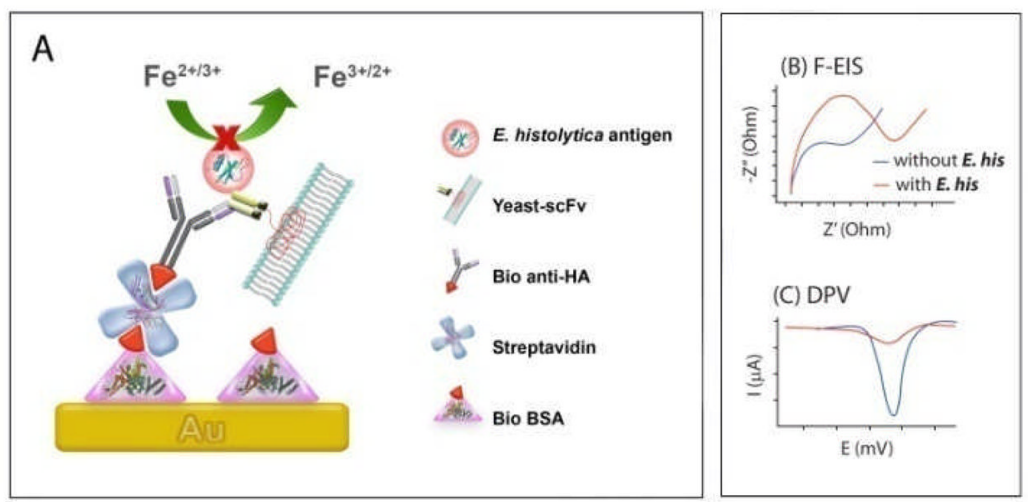

Fig. 1.

(A) Schematic of label-free protein detection. E. histolytica proteins complexed with surface attached yeast-scFv fragments which hinders the interfacial electron transfer reaction of the $\left[\mathrm{Fe}(\mathrm{CN})_{6}\right]^{3-/ 4-}$ process. (B) and $(\mathrm{C})$ represent hypothetical sensor responses at the presence and absence of E. histolytica proteins for the label-free impedance and voltammetric readouts, respectively. 

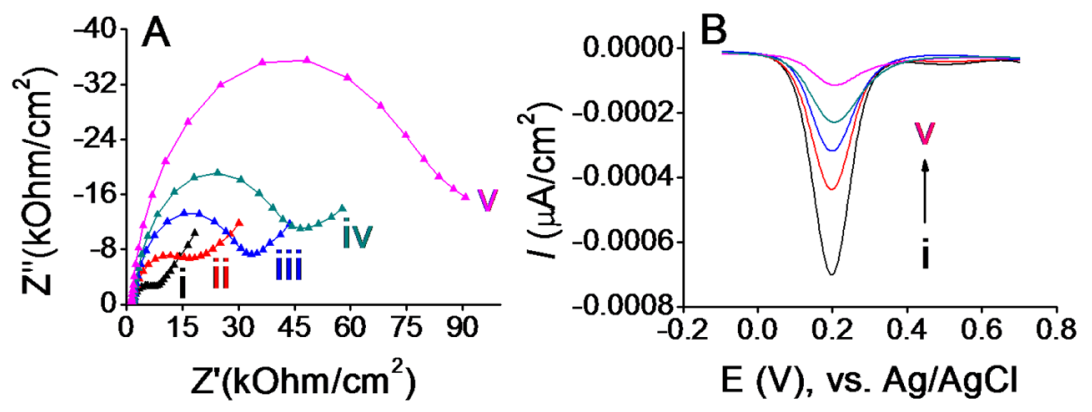

Fig. 2.

(A) Nyquist plots for (i) bare and modified electrodes for detecting (ii) bio BSA/ streptavidin, (iii) bio BSA/streptavidin/bio anti-HA, (iv) bio BSA/streptavidin/bio anti-HA/ yeast-scFv, and (v) bio BSA/streptavidin/bio anti-HA/yeast-scFv/'350' complex in $10 \mathrm{mM}$ phosphate buffer solution ( $\mathrm{pH} 7.4$ ) containing $2.5 \mathrm{mM} \mathrm{K}_{3}\left[\mathrm{Fe}(\mathrm{CN})_{6}\right], 2.5 \mathrm{mM} \mathrm{K}_{4}\left[\mathrm{Fe}(\mathrm{CN})_{6}\right]$ $\cdot 3 \mathrm{H}_{2} \mathrm{O}$, and $0.1 \mathrm{M} \mathrm{KCl}$. (B) Corresponding DPV responses. 

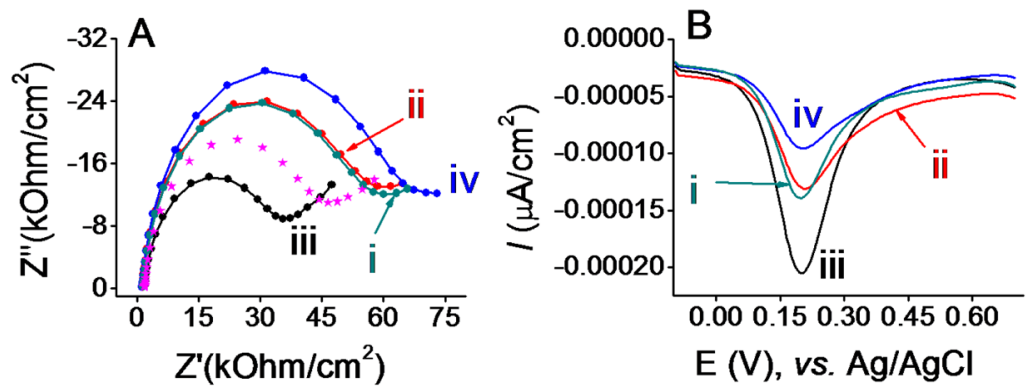

Fig. 3.

(A) Nyquist plots for immunosensing layer on gold electrodes for detecting (i) bio BSA/ streptavidin/bio anti-HA/yeast-scFv/Jacob, (ii) bio BSA/streptavidin/bio anti-HA/yeastscFv-2/'350', (iii) bio BSA/streptavidin/bio anti-HA/'350', and (iv) bio BSA/streptavidin/ bio anti-HA/yeast-scFv/'350' immunocomplex formation in $10 \mathrm{mM}$ phosphate buffer solution (pH 7.4) containing $2.5 \mathrm{mM} \mathrm{K}_{3}\left[\mathrm{Fe}(\mathrm{CN})_{6}\right], 2.5 \mathrm{mM} \mathrm{K}_{4}\left[\mathrm{Fe}(\mathrm{CN})_{6}\right] \cdot 3 \mathrm{H}_{2} \mathrm{O}$, and $0.1 \mathrm{M}$ $\mathrm{KCl}$. Protein concentrations for (i), (ii), (iii) and (iv) were 500, 500, 500, and $10 \mathrm{pg} / \mathrm{mL}$. Dotted line spectrum represents impedance at bio BSA/streptavidin/bio anti-HA/yeast-scFv sensing layer in the same electrolyte solution. (B) Corresponding DPV responses. 


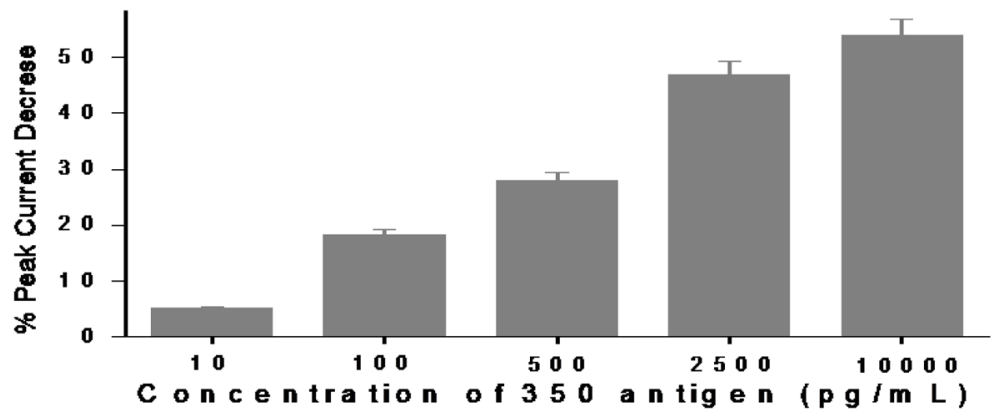

Fig. 4.

Change of the DPV responses at the immunosensing surface after incubation with designated concentrations of E. histolytica '350' antigen. 